\title{
Some Recent Results on Integrable Bilinear Equations
}

\author{
Xing-Biao $\mathrm{HU}^{\dagger}$ and Hon-Wah $T A M^{\ddagger}$ \\ $\dagger$ State Key Laboratory of Scientific and Engineering Computing, \\ Institute of Computational Mathematics and Scientific Engineering Computing, \\ Academia Sinica, P.O. Box 2719, Beijing 100080, P.R. China \\ E-mail: hxb@lsec.cc.ac.cn \\ $\ddagger$ Department of Computer Science, Hong Kong Baptist University, \\ Kowloon Tong, Hong Kong, P.R. China \\ E-mail: tam@comp.hkbu.edu.hk
}

\begin{abstract}
This paper shows that several integrable lattices can be transformed into coupled bilinear differential-difference equations by introducing auxiliary variables. By testing the Bäcklund transformations for this type of coupled bilinear equations, a new integrable lattice is found. By using the Bäcklund transformation, soliton solutions are obtained. By the dependent variable transformation, this new coupled bilinear equations can be reduced to a coupled extended Lotka-Voltera equation and another equation.
\end{abstract}

The purpose of this short paper is to search for new integrable systems in bilinear form. The methods used here are Hirota's method and Bäcklund transformations [1-7]. As we know, the use of the Hirota bilinear transformation in the search of exact solutions of continuous and discrete systems is now well established [1-5]. More recently, Hirota's method has been systematically used in the search for new integrable equations in both $(1+1)$ and $(2+1)$ dimensions by testing multi-soliton solutions or Bäcklund transformations (see, e.g. $[8,9]$ ). The key points behind these ideas to obtain new integrable systems are to first generalize bilinear forms of known integrable systems and then to test the generalized bilinear forms for multi-soliton solutions or Bäcklund transformations.

In this paper, we will focus on the differential-difference case and find some new integrable systems by testing their bilinear Bäcklund transformations. In order to do so, let us first recall the bilinear forms for some known integrable differential-difference equations.

Example 1. The so-called Belov-Chaltikian lattice is given by [10]

$$
\begin{aligned}
& b_{t}(n)=b(n)(b(n+1)-b(n-1))-c(n)+c(n-1), \\
& c_{t}(n)=c(n)(b(n+2)-b(n-1)) .
\end{aligned}
$$

By the dependent variable transformation

$$
b(n)=\left(\ln \frac{f\left(n+\frac{1}{2}\right)}{f\left(n-\frac{1}{2}\right)}\right)_{t}, \quad c(n)=\frac{f\left(n+\frac{5}{2}\right) f\left(n-\frac{3}{2}\right)}{f\left(n+\frac{3}{2}\right) f\left(n-\frac{1}{2}\right)},
$$

Copyright (C) 2001 by $X-B$ Hu and $H-W$ Tam 
equations (1) and (2) are transformed into the following bilinear form [11]

$$
\begin{aligned}
& \left(D_{t}^{2} e^{\frac{1}{2} D_{n}}-D_{z} e^{\frac{1}{2} D_{n}}\right) f(n) \bullet f(n)=0, \\
& \left(D_{z} e^{D_{n}}-D_{t}^{2} e^{D_{n}}+2 e^{2 D_{n}}-2 e^{D_{n}}\right) f(n) \bullet f(n)=0,
\end{aligned}
$$

with $z$ being an auxiliary variable, here the Hirota's bilinear differential operator $D_{x}^{m} D_{t}^{k}$, the bilinear difference operator $\exp \left(\delta D_{n}\right)$ and the bilinear differential-difference operator $D_{x}^{m} D_{t}^{k} \exp \left(\delta D_{n}\right)$ are defined by $[1-5]$

$$
\begin{aligned}
& \left.D_{x}^{m} D_{t}^{k} a \bullet b \equiv\left(\frac{\partial}{\partial x}-\frac{\partial}{\partial x^{\prime}}\right)^{m}\left(\frac{\partial}{\partial t}-\frac{\partial}{\partial t^{\prime}}\right)^{k} a(x, t) b\left(x^{\prime}, t^{\prime}\right)\right|_{x^{\prime}=x, t^{\prime}=t}, \\
& \left.\exp \left(\delta D_{n}\right) a(n) \bullet b(n) \equiv \exp \left[\delta\left(\frac{\partial}{\partial n}-\frac{\partial}{\partial n^{\prime}}\right)\right] a(n) b\left(n^{\prime}\right)\right|_{n^{\prime}=n}=a(n+\delta) b(n-\delta), \\
& D_{x}^{m} D_{t}^{k} \exp \left(\delta D_{n}\right) a(n) \bullet b(n) \\
& \left.\quad \equiv\left(\frac{\partial}{\partial x}-\frac{\partial}{\partial x^{\prime}}\right)^{m}\left(\frac{\partial}{\partial t}-\frac{\partial}{\partial t^{\prime}}\right)^{k} a(n+\delta, x, t) b\left(n-\delta, x^{\prime}, t^{\prime}\right)\right|_{x^{\prime}=x, t^{\prime}=t} .
\end{aligned}
$$

Example 2. Consider the Blaszak-Marciniak lattice [12]

$$
\begin{aligned}
& a_{t}(n)=c(n+1)-c(n-1), \\
& b_{t}(n)=a(n-1) c(n-1)-a(n) c(n), \\
& c_{t}(n)=c(n)(b(n)-b(n+1)) .
\end{aligned}
$$

By the dependent variable transformation

$$
a(n)=\frac{D_{t}^{2} f(n+1) \bullet f(n+1)}{2 f(n) f(n+2)}, \quad b(n)=\left(\ln \frac{f(n)}{f(n+1)}\right)_{t}, \quad c(n)=\frac{f(n) f(n+2)}{f^{2}(n+1)},
$$

equations (5), (6) and (7) are transformed into the following bilinear form [13]

$$
\begin{aligned}
& \left(D_{t}^{2}-2 D_{z} e^{D_{n}}\right) f(n) \bullet f(n)=0, \\
& \left(D_{z} D_{t}-4 \sinh ^{2}\left(\frac{1}{2} D_{n}\right)\right) f(n) \bullet f(n)=0,
\end{aligned}
$$

where $z$ is an auxiliary variable.

Example 3. The relativistic Toda lattice of Ruijsenaars is given by [14, 15]

$$
\ddot{q}_{n}=\dot{q}_{n}\left(\dot{q}_{n-1} \frac{g^{2} \exp \left(q_{n-1}-q_{n}\right)}{1+g^{2} \exp \left(q_{n-1}-q_{n}\right)}-\dot{q}_{n+1} \frac{g^{2} \exp \left(q_{n}-q_{n+1}\right)}{1+g^{2} \exp \left(q_{n}-q_{n+1}\right)}\right) .
$$

By the dependent variable transformation $q_{n}=\kappa \log \left(-g^{2}\right)+\log \frac{f_{n}}{f_{n+1}},(10)$ can be transformed into the trilinear form [16],

$$
\left|\begin{array}{ccc}
\dot{f}_{n-1} & f_{n-1} & f_{n} \\
\dot{f}_{n} & f_{n} & f_{n+1} \\
\ddot{f}_{n} & \dot{f}_{n} & \dot{f}_{n+1}
\end{array}\right|=0,
$$


which can be equivalently written as

$$
\begin{aligned}
& \left(D_{t}^{2} f_{n} \bullet f_{n}\right) \sinh ^{2}\left(\frac{D_{n}}{2}\right) f_{n} \bullet f_{n} \\
& \quad-\cosh \left(\frac{D_{n}}{2}\right)\left[D_{t} \exp \left(\frac{D_{n}}{2}\right) f_{n} \bullet f_{n}\right] \bullet\left[D_{t} \exp \left(\frac{D_{n}}{2}\right) f_{n} \bullet f_{n}\right]=0 .
\end{aligned}
$$

By introducing an auxiliary variable $z$, equation (12) can be decoupled into the bilinear form

$$
\begin{aligned}
& \left(D_{t}^{2} e^{\frac{1}{2} D_{n}}-D_{z} e^{\frac{1}{2} D_{n}}\right) f(n) \bullet f(n)=0 \\
& \left(D_{z} e^{D_{n}}-\frac{1}{2} D_{t}^{2} e^{D_{n}}-\frac{1}{2} D_{t}^{2}\right) f(n) \bullet f(n)=0 .
\end{aligned}
$$

Example 4. We now consider three new lattices. The first one is [17]

$$
\begin{aligned}
& u_{t t}(n+1)+u_{t t}(n)+u_{t t}(n-1)-3 u(n)\left(u_{t}(n+1)+u_{t}(n-1)\right) \\
& \quad+3 u(n+1) u_{t}(n+1)+3 u(n-1) u_{t}(n-1)-\frac{1}{4} u(n+1)-\frac{1}{4} u(n-1) \\
& \quad+\frac{1}{2} u(n)+[u(n+1)-2 u(n)+u(n-1)]\left[(u(n+1)-u(n-1))^{2}\right. \\
& \quad-(u(n+1)-u(n))(u(n)-u(n-1))]=0 .
\end{aligned}
$$

By the dependent variable transformation $u(n)=(\ln f(n))_{t}$, equation $(15)$ can be transformed into the bilinear form

$$
\begin{aligned}
& \left(D_{t}^{2} e^{\frac{1}{2} D_{n}}-D_{z} e^{\frac{1}{2} D_{n}}\right) f(n) \bullet f(n)=0, \\
& \left(D_{t}^{3} e^{\frac{1}{2} D_{n}}+3 D_{t} D_{z} e^{\frac{1}{2} D_{n}}-D_{t} e^{\frac{1}{2} D_{n}}\right) f(n) \bullet f(n)=0 .
\end{aligned}
$$

The second lattice under consideration is given by [18]

$$
\begin{gathered}
u_{t t t}(n+1)+u_{t t t}(n)+2\left(u_{t}(n+1)-u_{t}(n)\right)\left(u_{t t}(n+1)-u_{t t}(n)\right) \\
=e^{u(n+2)-2 u(n+1)+u(n)}-e^{u(n+1)-2 u(n)+u(n-1)} .
\end{gathered}
$$

By the dependent variable transformation $u(n)=\ln f(n)$, equation (18) can be transformed into the bilinear form

$$
\begin{aligned}
& \left(D_{t}^{2} e^{\frac{1}{2} D_{n}}-D_{z} e^{\frac{1}{2} D_{n}}\right) f(n) \bullet f(n)=0, \\
& \left(D_{z} D_{t}-4 \sinh ^{2} \frac{1}{2} D_{n}\right) f(n) \bullet f(n)=0 .
\end{aligned}
$$

The third lattice is given by [19]

$$
u_{t t}(n)+u_{t}(n)\left(u_{t}(n-1)-u_{t}(n+1)\right)=4 e^{u(n+2)-u(n-1)}-4 e^{u(n+1)-u(n-2)} .
$$


By the dependent variable transformation $u(n)=\ln (f(n+1) / f(n))$, equation (21) can be transformed into the bilinear form

$$
\begin{aligned}
& \left(D_{z} D_{t}-2 e^{D_{n}}+2\right) f(n) \bullet f(n)=0, \\
& \left(D_{z}^{3} D_{t}+6 D_{z}^{2} e^{D_{n}}+6 D_{z}^{2}-2 e^{D_{n}}+2\right) f(n) \bullet f(n)=0 .
\end{aligned}
$$

Based on these examples, it is quite natural for us to consider the following generalized bilinear equations

$$
\begin{aligned}
& F_{1}\left(D_{t}, D_{z}, \sinh \left(\alpha_{1} D_{n}\right), \ldots, \sinh \left(\alpha_{l} D_{n}\right)\right) f(n) \bullet f(n)=0, \\
& F_{2}\left(D_{t}, D_{z}, \sinh \left(\alpha_{1} D_{n}\right), \ldots, \sinh \left(\alpha_{l} D_{n}\right)\right) f(n) \bullet f(n)=0,
\end{aligned}
$$

and further search for new integrable differential-difference systems of this type by testing Bäcklund transformations, where $F_{i}(i=1,2)$ are two even order polynomials in $D_{t}, D_{z}$, $\sinh \left(\alpha_{1} D_{n}\right), \ldots$ and $\sinh \left(\alpha_{l} D_{n}\right)$, and $l$ is a given positive integer; the $\alpha_{i}, i=1,2, \ldots, l$, are $l$ different constants, and $F_{i}(0,0, \ldots, 0)=0$. We could search for new integrable systems of the type (24) and (25) via the following steps. Firstly following [9], we seek new bilinear forms $F_{1}$ and $F_{2}$ individually by testing Bäcklund transformations. If a Bäcklund transformation for $F_{1}=0$ is compatible with a Bäcklund transformation found for $F_{2}=0$, then this coupled system is also integrable. For example, from [9] we know that

$$
\left(D_{z} D_{t}+A D_{t} \sinh \left(D_{n}\right)-4 \sinh ^{2}\left(\frac{1}{2} D_{n}\right)\right) f(n) \bullet f(n)=0
$$

has a Bäcklund transformation where $A$ is an arbitrary constant. We also find that

$$
\left(D_{z} D_{t} e^{\frac{1}{2} D_{n}}+B D_{z} e^{\frac{1}{2} D_{n}}+C D_{t} e^{\frac{1}{2} D_{n}}\right) f(n) \bullet f(n)=0
$$

is integrable in the sense of having a Bäcklund transformation where $B$ and $C$ are arbitrary constants. After some trial and error, we know that (26) and (27) have compatible Bäcklund transformations if we choose $A=2, B=-1 / 2, C=0$. In this case, we have the following new coupled integrable bilinear system

$$
\begin{aligned}
& \left(D_{z} D_{t}+2 D_{t} \sinh \left(D_{n}\right)-4 \sinh ^{2}\left(\frac{1}{2} D_{n}\right)\right) f(n) \bullet f(n)=0, \\
& \left(D_{z} D_{t} e^{\frac{1}{2} D_{n}}-\frac{1}{2} D_{z} e^{\frac{1}{2} D_{n}}\right) f(n) \bullet f(n)=0,
\end{aligned}
$$

where $z$ is an auxiliary variable. Concerning (28) and (29), we have the following result:

Proposition. The system of bilinear Eqs. (28)-(29) has the following Bäcklund transformation:

$$
\begin{aligned}
& \left(D_{z}+\lambda^{-1} e^{-D_{n}}-\lambda e^{D_{n}}+\mu\right) f(n) \bullet g(n)=0, \\
& \left(D_{t} e^{-\frac{1}{2} D_{n}}+\lambda D_{t} e^{\frac{1}{2} D_{n}}-(\lambda-\lambda \gamma) e^{\frac{1}{2} D_{n}}+\gamma e^{-\frac{1}{2} D_{n}}\right) f(n) \bullet g(n)=0, \\
& \left(D_{z} e^{\frac{1}{2} D_{n}}-\lambda^{-1} D_{z} e^{-\frac{1}{2} D_{n}}+\lambda \omega e^{\frac{1}{2} D_{n}}-\omega e^{-\frac{1}{2} D_{n}}\right) f(n) \bullet g(n)=0,
\end{aligned}
$$

where $\lambda, \mu, \gamma$ and $\omega$ are arbitrary constants. 
This result can be proved by using Hirota's bilinear operator identities. Due to limited space, we do not give the details of the proof. Instead we are going to construct soliton solutions of (28)-(29) by using the BT (30)-(32). Firstly, by applying the BT (30)-(32) to the trivial solution $f(n)=1$, we can obtain the 1 -soliton solution

$$
g(n)=1+\exp \left(p n+2 \sinh (p) z+\frac{1}{2} \tanh \left(\frac{1}{2} p\right) t+\eta^{0}\right)
$$

where $p$ and $\eta^{0}$ are constants, for the parameters $\lambda=1, \mu=0, \gamma=\frac{1}{2}$ and $\omega=2 \sinh p$. Further by applying the BT (30)-(32) to the 1-soliton solution $f(n)=1+\exp \left(\eta_{1}\right)$ we can deduce the following 2-soliton solution

$$
g(n)=1+A_{1} e^{\eta_{1}}+e^{\eta_{2}}+A_{2} e^{\eta_{1}+\eta_{2}},
$$

where

$$
\begin{aligned}
& \eta_{i}=p_{i} n+2 \sinh \left(p_{i}\right) z+\frac{1}{2} \tanh \left(\frac{1}{2} p_{i}\right) t+\eta_{i}^{0}, \\
& A_{1}=\frac{\sinh \left(p_{1}\right)+\sinh \left(p_{2}\right)}{-\sinh \left(p_{1}\right)+\sinh \left(p_{2}\right)}, \quad A_{2}=-\frac{\sinh \left(\frac{1}{2}\left(p_{1}-p_{2}\right)\right)}{\sinh \left(\frac{1}{2}\left(p_{1}+p_{2}\right)\right)},
\end{aligned}
$$

with $p_{i}$ and $\eta_{i}^{0}$ constants for the set of parameters $\lambda=1, \mu=0, \gamma=\frac{1}{2}$ and $\omega=$ $2 \sinh \left(p_{2}\right)$. Besides, by using MATHEMATICA, we can show that (28)-(29) have the 3 -soliton solutions

$$
f=1+e^{\eta_{1}}+e^{\eta_{2}}+e^{\eta_{3}}+A_{12} e^{\eta_{1}+\eta_{2}}+A_{13} e^{\eta_{1}+\eta_{3}}+A_{23} e^{\eta_{2}+\eta_{3}}+A_{12} A_{23} A_{13} e^{\eta_{1}+\eta_{2}+\eta_{3}},
$$

where

$$
\begin{aligned}
& \eta_{i}=p_{i} n+2 \sinh \left(p_{i}\right) z+\frac{1}{2} \tanh \left(\frac{1}{2} p_{i}\right) t+\eta_{i}^{0}, \\
& A_{i j}=\frac{\sinh \left(\frac{1}{2}\left(p_{i}-p_{j}\right)\right)\left(\sinh \left(p_{i}\right)-\sinh \left(p_{j}\right)\right)}{\sinh \left(\frac{1}{2}\left(p_{i}+p_{j}\right)\right)\left(\sinh \left(p_{i}\right)+\sinh \left(p_{j}\right)\right)} .
\end{aligned}
$$

By the dependent variable transformation $u(n)=\ln f(n), v(n)=\frac{f_{z}(n)}{f(n)},(28)$ and (29) are transformed into the system

$$
\begin{aligned}
& \left(u_{t}(n+1)-u_{t}(n-1)-1\right) e^{u(n+1)+u(n-1)-2 u(n)}+1+v_{t}(n)=0, \\
& v_{t}(n+1)+v_{t}(n)+(v(n+1)-v(n))\left(u_{t}(n+1)-u_{t}(n)-\frac{1}{2}\right)=0 .
\end{aligned}
$$

In particular, if we choose $v(n)=0$ and $a(n)=e^{u(n)-u(n-1)}$, then (33)-(34) become an extended Lotka-Volterra lattice $[20,21]$

$$
\frac{d}{d t}(a(n) a(n+1))=a(n)(a(n+1)-a(n)) .
$$

Therefore the system (33) and (34) is a coupled extended Lotka-Volterra equation. We now derive the $z$-flow. In order to do so, we view $t$ appearing in (28)-(29) as an auxiliary 
variable. Setting $U(n)=\ln f(n), V(n)=\frac{f_{t}(n)}{f(n)}$, then (28)-(29) can be transformed into the following system in nonlinear variables

$$
\begin{aligned}
& (V(n)-V(n+2)+1) e^{U(n+2)+U(n)-2 U(n+1)} \\
& \quad+(V(n-1)-V(n+1)+1) e^{U(n+1)+U(n-1)-2 U(n)}-2 \\
& \quad+\left(V(n+1)-V(n)-\frac{1}{2}\right)\left(U_{z}(n+1)-U_{z}(n)\right)=0, \\
& V_{z}(n)+(V(n+1)-V(n-1)-1) e^{U(n+1)+U(n-1)-2 U(n)}+1=0 .
\end{aligned}
$$

In summary, we have observed that several integrable lattices can be transformed into coupled bilinear differential-difference equations by introducing auxiliary variables. We propose a coupled generalized Hirota's bilinear equations. By testing Bäcklund transformations for such type of coupled bilinear equations, a new integrable lattice is found. By using the Bäcklund transformation, soliton solutions are obtained. By the dependent variable transformation, this new coupled bilinear equations can be reduced to a coupled extended Lotka-Voltera equation and another equation. Besides, by using the Bäcklund transformation with special Bäcklund parameters $\lambda=1, \mu=0, \gamma=\frac{1}{2}, \omega=0$, we can obtain rational solutions for the lattices (33)-(34) and (36)-(37). For example, starting from the trivial solution $f(n)=1$, we have the following polynomial solution of (28)-(29):

$$
g(n)=n+2 z+\frac{1}{4} t
$$

\section{Acknowledgements}

This work was supported by Hong Kong Baptist University grant FRG/97-98/II-100, the National Natural Science Foundation of China (Project no.19871082) and the Chinese Academy of Sciences. One of authors (XBH) would like to express his sincere thanks to the organizers M Bruschi, F Calogero, O Ragnisco and A Verganelakis for their invitation to attend the workshop NEEDS'99 and partial financial support.

\section{References}

[1] Hirota R, Exact Solution of the Korteweg-de Vries Equation for Multiple Collisions of Solitons, Phys. Rev. Lett., 1971, V.27,1192.

[2] Hirota R, Direct Methods in Soliton Theory, in Solitons, Editors R K Bullough and P J Caudrey, Springer, Berlin, 1980.

[3] Hirota R and Satsuma J, A Variety of Nonlinear Network Equations Generated from the Bäcklund Transformation for the Toda Lattice, Prog. Theor. Phys. Suppl., 1976, N 59, 64.

[4] Matsuno Y, Bilinear Transformation Method, Academic, New York, 1984.

[5] Nimmo J J C, Hirota's Method, in Soliton Theory, A Survey of Results, Editor A P Fordy, Manchester University Press, Manchester, 1990.

[6] Miura R M (Editor), Bäcklund Transformations, in Lecture Notes in Mathematics, Vol.515, Springer, Berlin, 1976.

[7] Rogers C and Shadwick W F, Bäcklund Transformations and Their Applications, Vol.161, in Mathematics in Science and Engineering, Academic Press, New York, 1982.

[8] Hietarinta J, A Search for Bilinear Equations Passing Hirota's Three-Soliton Condition, J. Math. Phys., 1987, V.28, 1732. 
[9] Hu X-B, Clarkson P A and Bullough R, New Integrable Differential-Difference Systems, J. Phys. A: Math. Gen., 1997, V.30, L669-L676.

[10] Belov A A and Chaltikian K D, Lattice Analogues of W-algebras and Classical Integrable Equations, Phys. Lett. B, 1993, V.309, 268-274.

[11] Hu X-B and Zhu Z-N, A Bäcklund Transformation and Nonlinear Superposition Formula for the Belov-Chaltikian Lattice, J. Phys. A: Math. Gen., 1998, V.31, 4755-4761.

[12] Blaszak M and Marciniak K, r-matrix Approach to Lattice Integrable Systems, J. Math. Phys., 1994, V.35, 4661-4682.

[13] Hu X-B and Zhu Z-N, Some New Results on the Blaszak-Marciniak Lattice: Bäcklund Transformation and Nonlinear Superposition Formula, J. Math. Phys., 1998, V.39, 4766.

[14] Ruijsenaars S N M, Relativistic Toda Systems, Comm. Math. Phys., 1990, V.133, 217-247.

[15] Bruschi M and Ragnisco O, Recursion Operator and Bäcklund Transformations for the Ruijsenaars-Toda Lattice, Phys. Lett. A, 1988, V.129, 21-25; Lax Representation and Complete Integrability for the Periodic Relativistic Toda Lattice, 1989, V.134, 365-370.

[16] Hietarinta J and Satsuma J, The Trilinear Equation as a $(2+2)$-Dimensional Extension of the (1+1)-Dimensional Relativistic Toda Lattice, Phys. Lett. A, 1991, V.161, 267-273.

[17] Hu X-B and Wu Y-T, Application of the Hirota Bilinear Formalism to a New Integrable Differential-Difference Equation, Phys. Lett. A, 1998, V.246, 523-529.

[18] Wu Y-T and Hu X-B, A New Integrable Differential-Difference System and Its Explicit Solutions, J. Phys. A: Math. Gen., 1999, V.32, 1515-1521.

[19] Hu X-B, Wu Y-T and Geng X-G, Hirota Bilinear Approach to a New Integrable DifferentialDifference System, J. Math. Phys., 1999, V.40, 2001-2010; 1999, V.40, 4180.

[20] Narita K, Soliton Solution to Extended Volterra Equation, J. Phys. Soc. Japan, 1982, V.51, $1682-1685$.

[21] Hu X-B and Bullough R, Bäcklund Transformation and Nonlinear Superposition Formula of an Extended Lotka-Volterra Equation, J. Phys. A: Math. Gen., 1997, V.30, 3635-3641. 